\title{
The Continuous Strength Method of Cold-formed Stainless Steel Equal-leg Angle Section Stubs
}

\author{
Jing Yang ${ }^{1}$, Zhoupeng Cai ${ }^{2}$, Hongdong $\operatorname{Ran}^{2,}$ * \\ ${ }^{1}$ Xi' an Pengcheng Blasting Engineering Co. Ltd, Xi'an, China \\ ${ }^{2}$ School of Civil Engineering, University of Archetechtrue and Technology, Xi'an, China
}

\section{Email address:}

15109257624@163.com (Jing Yang),1014466935@qq.com (Zhoupeng Cai), ranhd@163.com (Hongdong Ran)

*Corresponding author

\section{To cite this article:}

Jing Yang, Zhoupeng Cai, Hongdong Ran. The Continuous Strength Method of Cold-formed Stainless Steel Equal-leg Angle Section Stubs. Science Discovery. Vol. 7, No. 5, 2019, pp. 323-329. doi: 10.11648/j.sd.20190705.21

Received: August 20, 2019; Accepted: November 14, 2019; Published: November 18, 2019

\begin{abstract}
Structural stainless steel requires appropriate recognition of its beneficial properties such as material nonlinearity and significant strain hardening. The recently proposed Continuous Strength Method (CSM) exploits those benefits through a strain based approach for both stocky and slender cross-sections, and is shown to yield a higher level of accuracy and consistency, as well as design efficiency, in the capacity predictions of stainless steel cross-sections. Although there have been extensive and comprehensive studies on SHS, RHS, round tubes and H-sections stubs, but research into cold-formed stainless steel equal-leg angle section stubs remains scarce. In this paper, the scope of the CSM is extended to cover the design of cold-formed stainless steel equal-leg angle section stubs. Developed FE models included material nonlinearities as well as initial geometric imperfections. A comprehensive parametric study has been carried out covering a wide range of slenderness with different cross section geometries for the considered angle stubs. Cross-section resistances obtained from the numerical study were used to assess the performance of the current Continuous Strength Method (DSM) guidelines and EC3 when applied for stainless steel equal-leg angle section stubs; obtained comparisons showed considerable conservatism. A modified design method for cold-formed stainless steel equal-leg angle section stubs is proposed herein following CSM techniques, which provides considerably more accurate predictions for the considered cold-formed stubs. Reliability of the proposed design equations is also presented showing a good agreement with both experimentally and numerically obtained results.
\end{abstract}

Keywords: Stainless Steel, Cold-formed, Equal-leg Angle, The Continuous Strength Method, Cross Section Strength

\section{不锈钢冷弯等肢角钢短柱的连续强度法}

杨靖 ${ }^{1}$, 蔡洲鹏 ${ }^{2}$, 再红东 ${ }^{2 *}$

1西安鹏程爆破工程有限公司, 西安, 中国

2西安建筑科技大学土木工程学院, 西安, 中国

\section{邮箱}

15109257624@163.com（杨靖），1014466935@qq.com（蔡洲鹏）, ranhd@163.com（申红东）

摘要: 结构不锈钢设计中需要适当利用其诸如材料非线性和应变硬化等优点, 最近提出的连续强度法(CSM)可实现此 目的且计算效率高。虽然该方法已应用于细长和非细长截面的承载力计算, 但仅适用于方管、矩管、圆管及 $\mathrm{H}$ 型截面。 本文将此方法扩展到冷弯等肢角钢截面。进行了一项全面的有限元研究, 以补充有限数量的可用试验结果。得到的有 限元结果用于提出不锈钢冷弯等肢角钢的截面承载力设计方法。将所提出方法与EC3指南计算结果进行比较, 比较结 果表明该方法与FE结果吻合良好, 且可靠性好。 
关键词: 不锈钢, 冷成型, 等肢角钢, 连续强度法, 截面承载力

\section{1. 引言}

由于耐腐蚀性好、强度高、维护成本低和美观等优异 材料性能, 不锈钢合金越来越多地用于建筑结构中。尽管 比起传统碳钢具有明显优势, 但由于缺乏恰当的设计方法 而不能充分发挥其优异性能, 因此不锈钢在结构中的应用 受到限制。

虽然不锈钢具有与碳钢明显不同的非线性应力-应变 关系, 但基于碳钢理想弹塑性材料模型提出的有效宽度法 是各国不锈钢设计规范处理不锈钢截面局部屈曲的方法。 SEI/ASCE-8[1]和AS/NZS4673[2]给出的截面长细比限值 为 0.673 , 大于该值时必须考虑有效截面。欧洲规范 3(EC3)[3] 将不锈钢截面分为四个级别, 其中4级是细长截 面。中国规范CECS-410[4]虽未对截面分类，但对所有设 计截面必须判断是否全截面有效。有效宽度法中, 毛截面 面积减小为有效截面, 且需基于有效尺寸重新计算所有几 何特性, 以考虑局部屈曲引起的承载力降低。有效截面几 何特性计算过程繁复, 且未能考虑不锈钢材料的非线性应 力-应变关系及应变硬化的有利影响, 因此直接将此方法 应用于不锈钢构件设计并不合理。

最近提出的连续强度法(CSM) 不对截面分类且通过 连续设计曲线利用材料应变硬化的有利影响。Gardner和 Nethercot[5,6]首先将该方法应用于不锈钢管截面。Gardner 和Ashraf[7]将这种方法扩展到其他非线性金属材料, 如铝 和高强度钢, 结果表明该方法具有普遍适用性, Ashraf等 [8]提出了不锈钢钢管和开口截面 “基于变形能力的承载力” 的设计方法。Gardner[9]首先提出术语“连续强度法”, 并 使该方法更便于使用。最近, Afshan和Gardner[10]使用一 种简单的线弹性硬化材料模型提出了CSM设计指南, 采用 此材料模型时, 非细长截面(即, 截面长细比 $\bar{\lambda}_{p} \leq 0.68$ ) 的截面承载力计算结果准确且离散性小。这一修正使CSM 可以更直接的利用不锈钢材料应变硬化的有利影响。

由于细长截面在实际工程中应用非常普遍, 简单的设 计方法将推进其在结构中的应用。Ahmed和Ashraf[11]在 可应用于紧凑截面的连续强度法的基础上, 建立截面变形 能力与等效弹性变形能力之间的关系, 考虑了局部屈曲对 截面承载力的影响, 因而将CSM的应用范围扩大到细长截 面。至此, CSM 可用于准确计算不同截面等级的构件截面 承载力, 既考虑了材料的非线性应力应变关系及应变硬化 的有利影响, 又省去了有效截面几何特性计算的咒长过程, 简化了构件截面承载力的设计方法。

然而, 现有CSM仅针对如奥氏体、铁素体、双相体等 各种常见等级不锈钢制造的矩管截面(RHS)、方管截面 (SHS)和H形截面的受压和面内抗弯承载力进行了研究。对 于常用于工程中的等肢角钢截面研究甚少, 仅见Zhang和 $\operatorname{Tan}[12]$ 不锈钢冷弯等肢角钢柱进行试验和数值分析, 该研 究为此类型截面应用于工程实际提供了试验数据及数值 研究基础。
本研究根据可用的试验结果建立并验证了数值模型, 采用该模型计算得到大量不锈钢冷弯等肢角钢截面短柱 的结构性能数据。所有试验和数值结果用于评判欧洲欧规 范及现有CSM设计公式对不锈钢冷弯等肢角钢的适用性。 在对现有CSM的适用性进行判断后, 提出了适用于不锈钢 冷弯等肢角钢截面承载力计算的CSM方法。

总的来说, 对不锈钢冷弯等肢角钢截面, 本研究推荐 的CSM设计方法可得到准确、一致且可靠的截面承载力计 算结果。

\section{2. 不锈钢设计规范的截面承载力设计方法}

国际主流不锈钢设计规范包括美国规范[1]、澳大利亚 /新西兰规范[2]、欧洲规范[3]和中国规范[4]。欧洲规范经 过多次修订后是国际上最新的不锈钢设计规范。然而, 所 有这些设计规范都按照传统的有效宽度法来处理细长截 面的局部屈曲。

\section{1. 欧洲规范(EC3)}

与EN1993-1-1[13]中规定的碳钢分类相似, EC3[3]将 不锈钢截面分为四个不同级别, 每个级别具有不同板件长 细比限值。对于不同的截面分类, 提出了一系列板件宽厚 比 (b/t) 限值, 板件宽厚比限值取决于材料特性 $\varepsilon=\sqrt{\left(235 / \sigma_{0.2}\right) /(E / 210000)}$ 、边缘支承条件(即内部或 外伸板件)和施加的应力场形状。由于没有明确屈服点, $0.2 \%$ 弹性应力 $\left(\sigma_{0.2}\right)$ 被认为是不锈钢的屈服应力 $\left(\sigma_{y}\right)$ 。对 于受压截面, 1-3级和4级截面间有明确区别。1-3级截面被 认为是非细长或紧凑截面, 通常由于材料屈服和/或非弹 性局部屈曲破坏, 其承载力大于弹性承载力。而4级被认 为是细长截面, 在低于屈服应力的平均应力下局部屈曲可 能会导致构件破坏。

截面分类与构成截面的长细比最大的板件相关, 且未 考虑板件间的相互作用。一旦超过3级限值, 需用有效宽 度法计算有效截面, 以考虑在达到 $\sigma_{0.2}$ 之前发生的局部屈 曲的影响。因此, 截面抗压承载力 $N_{c, R d}$ 由式(1)或(2)确定, 其中 $A_{g}$ 是毛截面面积, $A_{\text {eff }}$ 是有效截面面积, $\gamma_{M 0}$ 是规 范中规定的安全系数。

值得注意的是，对截面分类并计算细长截面的有效截 面几何特性需要大量计算工作。

对 $1 、 2$ 和 3 级截面:

$$
N_{c, R d}=\frac{A_{g} \sigma_{0.2}}{\gamma_{M 0}}
$$

对4级截面:

$$
N_{c, R d}=\frac{A_{e f f} \sigma_{0.2}}{\gamma_{M 0}}
$$




\section{2. 连续强度法(CSM)}

\subsection{1. 简介}

连续强度法(CSM)[14-16]是一种基于变形的设计方 法, 最初提出的目的是设计不锈钢紧凑(即 1,2 和3级)截面 时可以合理利用材料的应变硬化。在轴压[17-20]荷载作用 下, 该方法对非细长不锈钢截面的承载力计算结果精度高。

\subsection{2. 非细长截面的 CSM}

非细长截面的CSM设计方法采用“基本曲线”来确定 在局部屈曲破坏前截面可以达到的最大应变, 同时采用线 弹性硬化材料模型可使设计应力大于屈服应力。基于回归 拟合一系列包括奥氏体、双相和铁素体不锈钢、碳钢、高 强钢和铝等金属材料的受压试验数据的基本曲线, 给出了 截面长细比 $\bar{\lambda}_{p}$ 和用应变比 $\left(\varepsilon_{c s m} / \varepsilon_{y}\right)$ 表示的变形能力间 的关系, 由公式(3)给出, 其中 $\varepsilon_{c s m}$ 是可达到的最大应变, $\varepsilon_{y}=\sigma_{y} / E$, 为屈服应变。公式(3)给出了应变比 $\left(\varepsilon_{c s m} / \varepsilon_{y}\right)$ 的两个限值: 第一个限值为 15 , 是防止过度变形, 也满足 EN1993-1-4[21]中给出的材料延性要求, 而与采用的线弹 性硬化材料模型相关的第二个限值是避免过高估计材料 强度。与直接强度法(DSM) 类似, 连续强度法 $(\mathrm{CSM})$ 也使 用截面长细比 $\bar{\lambda}_{p}$ 而不是单个板的长细比 $\bar{\lambda}_{l}$, 因此考虑了 板件间相互作用的有利影响。注意公式(3)定义的基本曲线, 适用于 $\bar{\lambda}_{p}$ 小于等于 0.68 的非细长截面。

$$
\frac{\varepsilon_{c s m}}{\varepsilon_{y}}=\frac{0.25}{\bar{\lambda}_{p}^{3.6}} \leq \min \left(15, \frac{C_{1} \varepsilon_{u}}{\varepsilon_{y}}\right) \text {, 当 } \bar{\lambda}_{p} \leq 0.68
$$

$\mathrm{CSM}$ 线弹性硬化材料模型具有四个材料参数 $\left(C_{1}\right.$ 、 $C_{2} 、 C_{3}$ 和 $\left.C_{4}\right)$, 应变硬化斜率 $E_{s h}$ 由公式(4)计算得到。 通过最小二乘回归, 基于材料拉伸试样数据校准每种金属 材料的参数值。 $\mathrm{CSM}$ 材料模型参数 $C_{1}$ 用于定义截止应变 (见公式(3)), 以确保材料强度不会从采用的线性硬化材料 模型中过度预测。CSM材料参数 $C_{2}$ 用于公式(4)中定义应 变硬化斜率 $E_{s h}$, 而参数 $\varepsilon_{u}=C_{3}\left(1-\sigma_{y} / \sigma_{u}\right)+C_{4}$ 为对应于 材料极限强度 $\sigma_{u}$ 的应变。

$$
E_{s h}=\frac{\sigma_{u}-\sigma_{y}}{C_{2} \varepsilon_{u}-\varepsilon_{y}}
$$

在确定最大可达应变 $\varepsilon_{c s m}$ 和应变硬化模量 $E_{s h}$ 后, CSM设计应力 $\sigma_{c s m}$ 可以从公式(5)计算得到, 然后用公式 (6)计算截面抗压承载力 $N_{c s m, R d}$, 其中 $\gamma_{M 0}$ 是截面承载力 安全系数, 不锈钢的推荐值为 1.1 。

$$
\begin{gathered}
\sigma_{c s m}=\sigma_{y}+E_{s h}\left(\varepsilon_{c s m}-\varepsilon_{y}\right) \\
N_{c s m, R d}=\frac{A \sigma_{c s m}}{\gamma_{M 0}}
\end{gathered}
$$

通过与试验和有限元结果的全面比较知, CSM对不锈 钢非细长截面的承载力计算精度高且离散性小, 由于考虑 了应变硬化的有利影响, 因此适用于不锈钢非细长截面在 轴心受压[17-21]时的截面承载力计算。

\subsection{3. 细长截面的 CSM}

在成功应用于不锈钢非细长截面后, CSM设计方法应 用到细长截面设计, 此类构件在达到 $0.2 \%$ 弹性极限应力 前发生局部屈曲, 且并未出现明显应变硬化现象。CSM通 常有两个主要组成部分: 一个是基本曲线, 该曲线定义了 在局部屈曲破坏前截面可以达到的最大应变, 另一个是线 弹性硬化材料模型, 该模型提供了不锈钢材料的非线性应 力-应变关系。而采用的 CSM材料模型基于对材料单向拉 伸试验数据的分析进行校准, 因此与截面长细比无关。因 此, 将CSM扩展到细长截面设计的关键步骤在于为长细比 $\bar{\lambda}_{p}>0.68$ 的细长截面建立第二条基础曲线。

通过回归拟合试验数据得到非细长截面的 CSM基本 曲线, 试验局部屈曲极限应变 $\varepsilon_{l b}$ 为极限荷载下的最大应 变 $\varepsilon_{l b}$, 等于短柱破坏时试件末端缩短 $\delta_{u}$ 与试件长度 $L$ 之 比。

然而, 对于在屈服前因局部屈曲而破坏的细长截面, 截面承载力受到弹性屈曲和屈曲后性能的影响, 因此基于 试验局部屈曲极限应变定义提出的基本曲线将导致所采 用的CSM材料模型对破坏应力的过高估计。

因此，等效局部屈曲应变[11]的概念用于细长截面试 验极限应变的定义, 以避免设计破坏应力的计算结果过大。 考虑到CSM采用直至屈服应力的线性应力-应变材料模型, 基于线性荷载变形的假设确定了低于屈服强度的细长截 面构件的等效试验局部屈曲极限应变 $\varepsilon_{l b, e}$; 因此细长截面 柱 $\varepsilon_{l b, e}=N_{u, t e s t} /(E A)$, 其中 $N_{u, t e s t}$ 为试验极限荷载。因此, 短柱的试验局部屈曲应变比(定义为等效局部屈曲变形与 屈服应变 $\varepsilon_{y}$ 之比)等于试验极限承载力与屈服承载力的比 值, 见公式(7), 其中 $N_{y}=A \sigma_{y}$ 为截面屈服荷载。此外, 与非细长截面的基本曲线不同[24], 采用这种变形能力的 定义在材料应力低于 $0.2 \%$ 弹性极限应力不需要进一步考 虑实际材料应力-应变关系与 CSM 线弹性材料模型间的差 异, 因此 $\varepsilon_{c s m} / \varepsilon_{y}=\varepsilon_{l b, e} / \varepsilon_{y}$ 。

$$
\varepsilon_{c s m} / \varepsilon_{y}=\varepsilon_{l b, e} / \varepsilon_{y}=N_{u, t e s t} / N_{y}
$$

收集以往短柱试验数据提出了 $\bar{\lambda}_{p}>0.68$ 的细长截面 的第二条基本曲线。为了与 $\mathrm{CSM}$ 的非细长截面基本曲线保 持一致, 细长截面的基本曲线应该通过细长和非细长截面 间的长细比限值(过渡点), 即 $(0.68,1)$ 。得到的细长截面 $\mathrm{CSM}$ 基本曲线见公式(8)。但应注意, 两条曲线在 $\bar{\lambda}_{p}=0.68$ 处连续但斜率不连续。

$$
\frac{\varepsilon_{c s m}}{\varepsilon_{y}}=\left(1-\frac{0.222}{\bar{\lambda}_{p}^{1.050}}\right) \frac{1}{\bar{\lambda}_{p}^{1.050}}
$$


用相应的屈服(弹性)应力乘以应变比可计算得到 CSM截面承载力, 见公式(9)。

$$
N_{c s m, R d}=\frac{\varepsilon_{c s m}}{\varepsilon_{y}} \frac{A \sigma_{0.2}}{\gamma_{M 0}}
$$

\section{3. 有限元模型及试验验证}

\section{1. 简介}

使用商业有限元分析软件包ABAQUS[22]进行有限 元模拟。首先根据试验结果验证有限元模型, 并研究各种 输入参数对有限元模型的影响。经过验证的有限元模型用 于进行参数分析, 得到的计算结果用于补充有限的试验数 据。

\section{2. 有限元模型基本假定}

按照[23-24]的方法建立考虑材料和几何非线性的有 限元模型。单元采用减缩积分四节点双曲壳单元S4R, 对 于冷弯角钢的平直段采用均匀网格, 网格尺寸不超过 $2 \mathrm{~mm}$, 截面角部划分 6 个单元, 这样可以兼顾计算精度和计算效 率。材料模型采用由Arrayago等[25]修正的Rasmussen[26] 两阶段Ramberg-Osgood(R-O)[27]模型。

在成形过程中, 由于发生塑性变形, 冷成型截面出现 材料强度提高的现象。这种现象在截面角部非常明显, 因 此, 在角部及与之相连平直段延伸 2 倍角钢肢厚范围内使 用角部材性单向拉伸试验结果 [28]。

有限元模型中短柱两端固定，仅允许柱顶发生纵向位 移。底部和顶部的所有节点分别与位于相应截面形心处的 两个参考点耦合。除了顶部参考点的坚向位移外, 约束两 个参考点的所有自由度以模拟短柱两端固定的边界条件。 通过对顶部参考点施加坚向位移为模型加载。

\section{3. 初始几何缺陷}

薄壁构件的性能受到初始几何缺陷的影响。因为在短柱 试验中均未观察到构件的整体屈曲, 因此在有限元分析中只 考虑了局部初始几何缺陷。有限元模型中使用特征值分析得 到的最低阶弹性屈曲模态来模拟试件的初始几何缺陷分布。

为了评估初始几何缺陷大小对模型精度的影响, 考虑 了三个初始几何缺陷大小: 1)由Gardner和Nethercot[29]改 进的Dawson和Walker模型[30], 2)t/100和3) $t / 10$, 其中 $t$ 是 角钢肢厚。然后使用改进的Riks方法[31-32]进行几何和材 料非线性分析, 该方法可以得到包括下降段的完整的短柱 荷载-变形曲线。

\section{4. 残余应力}

冷弯型截面在成形过程中由于不均匀的塑性变形产 生弯曲残余应力。然而, 由于膜残余应力对冷成型不锈钢
角钢的影响可忽略不计, 且在材性试验中已包含了厚度方 向的残余应力[33-34], 因此在有限模型中不考虑残余应力 的影响。

\section{5. 有限元模型验证}

通过比较从有限元模拟与相应试验得到的破坏荷载、 荷载-柱中部扭转角曲线和破坏模式来评估有限元模型的 正确性。

表1给出了三组初始几何缺陷大小FE与试验破坏荷载 的比值, 表明三组初始几何缺陷情况下计算结果的精度均 很高、离散性小。

表1 有限元计算结果与试验截面承载力比较。

\begin{tabular}{lllll}
\hline \multirow{2}{*}{ 试件截面 } & \multirow{2}{*}{ 试件编号 } & \multicolumn{3}{l}{ 初始几何缺陷 } \\
\cline { 3 - 5 } & D\&W模型 & $\mathbf{t} / \mathbf{1 0 0}$ & $\mathbf{t} / \mathbf{1 0}$ \\
\hline \multirow{2}{*}{ A50X6 } & A50X6-150 & 1.01 & 1.02 & 0.95 \\
& A50X6-150R & 1.01 & 1.03 & 0.94 \\
A100X6 & A100X6-300 & 0.93 & 0.98 & 0.89 \\
平均值 & A100X6-300R & 1.00 & 1.05 & 0.94 \\
变异系数 & & 0.99 & 1.02 & 0.93 \\
\hline
\end{tabular}

图1比较了典型不锈钢冷弯等肢角钢固端短柱试件 A50-150和A100-300的试验和 FE荷载-柱中部扭转角曲线, 可以看出FE曲线很好地模拟了相应试件的试验曲线。图中 给出的FE曲线为初始几何缺陷采用改进的 Dawson和 Walker模型的计算结果。由于[12]未给出短柱试件的破坏 模式, 但从分析可知, 角钢短柱在轴力作用下必然发生塑 性局部屈曲, 这与有限元分析结果吻合, 图2给出了发生 塑性局部屈曲的短柱试件A $100 \times 100 \times 6$ 的破坏模式。

总的来说, 有限元模型能够准确地模拟不锈钢冷弯等 肢角钢固端柱试件的试验响应, 因此可用于后续的参数研 究。

\section{4. 参数分析}

为补充有限的试验数据, 考虑更多截面长细比情况下 的构件截面承载力, 经验证的有限元模型用于一系列参数 研究。共分析了 470 个不同的冷弯等肢角钢截面, 角钢肢 宽在25-200mm间变化, 肢厚在2-6mm间变化, 相应的截 面长细比在0.25-3.0间变化。材料为奥氏体不锈钢(1.4420), 选自 [12]中的A $50 \times 50 \times 6$ 试件, 有限元模型截面平直段和角 部分别采用单向拉伸试验实测材料性能, 材料性能列于表 2 中。对于短柱, 每个模型的长度为截面最大尺寸的三倍。 改进的Dawson和Walker模型用于计算初始局部缺陷大小, 有限元模型中不考虑残余应力。有限元分析结果及文献 [12]中的试验数据用于评价现行欧洲规范(EC3)[3]及CSM 设计方法对不锈钢冷弯等肢角钢截面承载力的适用性。

表2 试验试件的材料性能[12]。

\begin{tabular}{llllll}
\hline 试件截面 & 取样位置 & $\mathbf{E}(\mathbf{G P a})$ & $\boldsymbol{\sigma}_{\mathbf{0 . 2}}(\mathbf{M P a})$ & $\boldsymbol{\sigma}_{\mathbf{u}}(\mathbf{M P a})$ & $\mathbf{R}-\mathbf{O}$ 参数(n) \\
\multirow{2}{*}{ A50X6 } & 平直段 & 198.2 & 278.2 & 637.0 & 6.8 \\
& 角部 & 187.1 & 619.4 & 880.5 & 2.2 \\
& & &
\end{tabular}




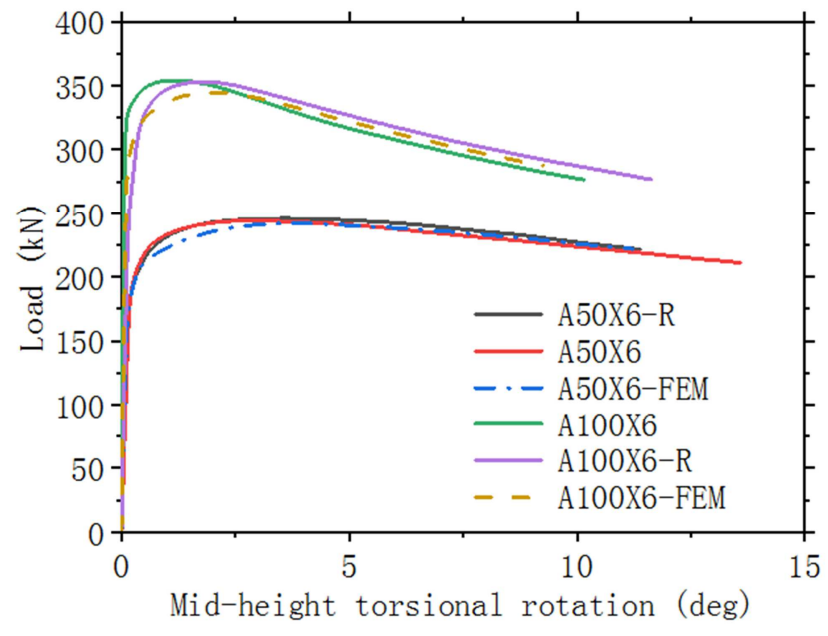

图1 试验试件[12]与有限元模型荷载-柱中转角关系曲线。

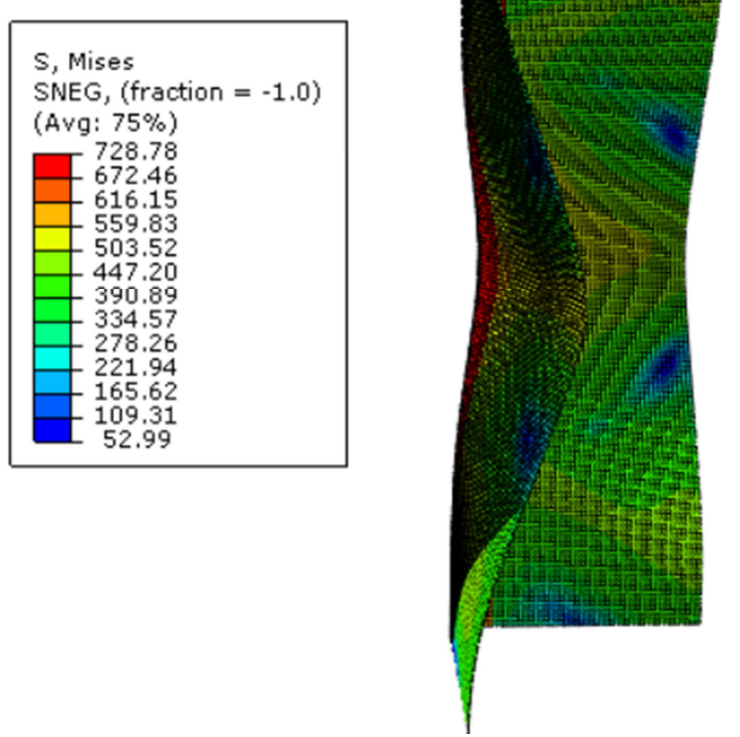

图2 试件A100×100×6的破坏模式。

\section{5. 欧洲规范 $(\mathrm{EC})$ 及 3 现有 CSM 设计方法评价}

通过将数值计算承载力 $\mathrm{N}_{\mathrm{u}}$ 与未考虑安全分项系数的 设计承载力 $\mathrm{N}_{\mathrm{u}, \text { pred }}$ 进行比较, 对两种设计方法进行定量评 价。图3给出了EC3 规范及现有CSM设计方法计算结果、 有限元及试验结果的比较。由图可知, 现有CSM方法及 EC3 方法计算结果均明显偏于保守。现有CSM方法比EC3 方法得到的平均承载力的计算结果更小, 但离散性小。现 有 CSM计算结果及EC3 计算结果与有限元结果之比的平 均值分别为 0.74 和 0.77 , 变异系数 $\mathrm{CoV}$ 分别为 0.09 和 0.12 。 比较结果表明, 现有CSM及EC3 设计方法均不能准确计算 不锈钢冷弯等肢角钢的截面承载力。

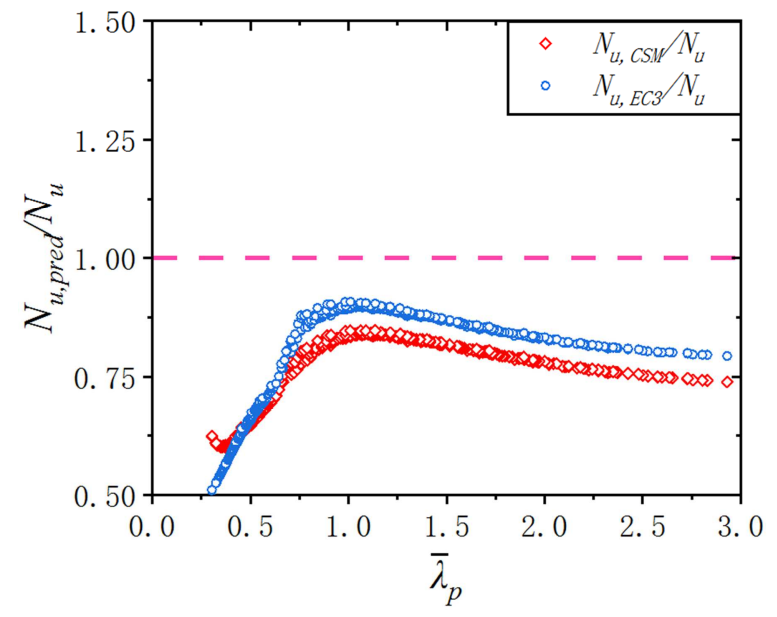

图3 欧洲规范及现有CSM设计方法计算结果与有限元计算结果比较。

\section{6. 推荐的冷弯不锈钢等肢角钢CSM设计方法}

根据公式(10)计算得到有限元计算得到的端部缩短 $\delta_{\mathrm{u}}$ 与试件长度 L之比, 相对截面长细比绘制于图4中, 由图可 知该曲线符合指数函数关系, 通过回归拟合有限元数据得 不锈钢冷弯等肢角钢截面的 CSM基本曲线, 见公式(11), 图4中也给出了公式(11)对应的CSM基本曲线, 式中还给 出了两个变形能力限制, 原因与现有CSM设计方法相同。

$$
\varepsilon_{c s m}=\varepsilon_{l b}-0.002=\frac{\delta_{u}}{L}-0.002
$$

有限元结果用于校准不锈钢冷弯等肢角钢截面承载 力的 CSM设计公式。建议将现有 CSM 公式(公式(3)和(8)) 修改为不锈钢冷弯等肢角钢截面承载力 CSM公式(公式 (11))。

$$
\begin{gathered}
\mathcal{E}_{c s m}=\frac{0.0013}{\bar{\lambda}_{p}^{0.73}} \text { 且 } \frac{\varepsilon_{c s m}}{\varepsilon_{y}} \leq \min \left(15, \frac{C_{1} \varepsilon_{u}}{\varepsilon_{y}}\right) \\
\sigma_{c s m}=\mathrm{E} \varepsilon_{c s m} \\
N_{c s m, R d}=\frac{\sigma_{c s m} A}{\gamma_{M 0}}
\end{gathered}
$$

公式(11)可适用于整个截面长细比范围, 由公式(11) 计算得到截面变形能力后, 由公式(12)计算CSM应力, 再 根据公式(13)得到不锈钢冷弯等肢角钢截面承载力, 该公 式使用更简单, 且在 $\bar{\lambda}_{p}=0.68$ 处曲线及曲线斜率均连续。 使用推荐的 CSM设计方法计算得到的名义构件承载力 $\mathrm{N}_{\mathrm{u}, \mathrm{pro}}$ 与由 FE模型得到的承载力 $\mathrm{N}_{\mathrm{u}}$ 进行比较。比较结果在 图5中给出。对于所有试件, $\mathrm{N}_{\mathrm{u}, \mathrm{pro}} / \mathrm{N}_{\mathrm{u}}$ 的平均值为 1.01 , 标 准偏差为 0.03 ; 可以看出, 本文推荐的CSM设计公式比现 行欧洲规范(EC3)及现有CSM设计公式计算结果更准确且 离散性更小。

然而从图中还可以看出, 按照推荐公式计算的不锈钢 冷弯等肢角钢截面承载力虽然和有限元分析结果吻合很 
好, 但仍有一部分结果和有限元计算结果的比值大于 1.0 , 表明按本文推荐的公式计算的截面承载力仍有一部分偏 于不安全。针对不锈钢冷弯等肢角钢截面承载力更精确的 CSM设计公式仍在进一步研究中。

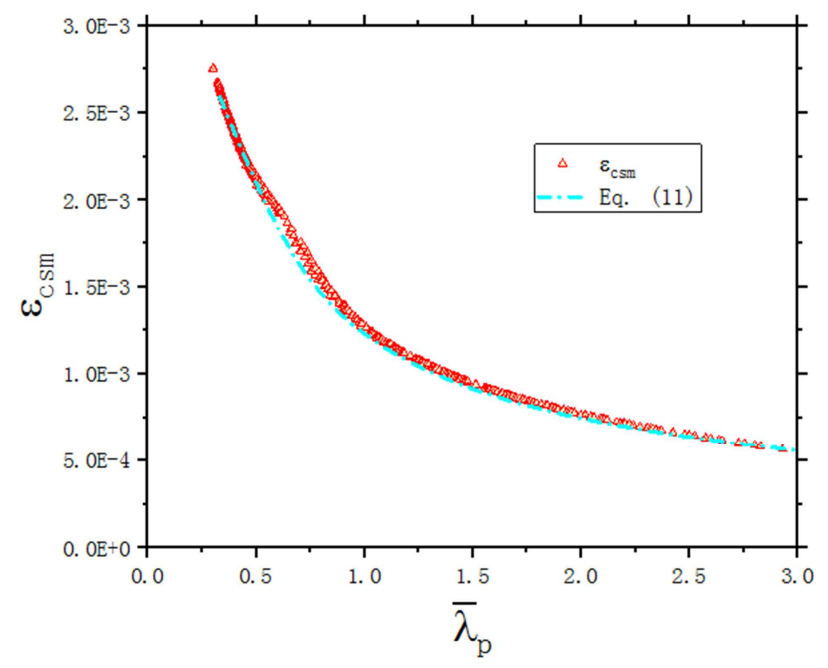

图4 试件变形能力与截面长细比关系。

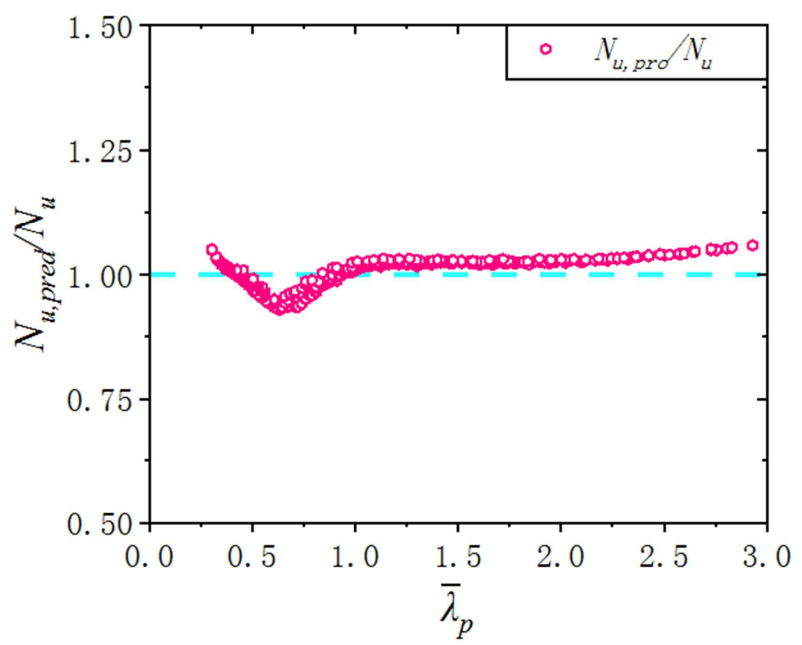

图5 推荐CSM设计方法计算结果与FE计算结果比值。

\section{7. 可靠性分析}

使用EN 1990 - Annex D[35]的方法进行标准统计分析 以评估本文推荐的CSM设计公式的可靠性。表3给出了分 析中使用的关键参数, 其中 $\mathrm{n}$ 是 $\mathrm{FE}$ 结果的总数, $k_{d, n}$ 是数 据数量 $\mathrm{n}$ 的设计值(极限状态)分位系数, $\mathrm{b}$ 是基于每组数据 的最小二乘拟合的FE结果与本文推荐公式计算结果的平 均比值。 $V_{\delta}$ 是相对于承载力模型的试验和 $\mathrm{FE}$ 模拟的变异 系数, $V_{r}$ 是包含模型和基本变量不确定性的组合变异系 数, $\gamma_{M 0}$ 是截面承载力的安全分项系数。根据[36]的建议, 奥氏体不锈钢的材料超强系数为 1.3 , 材料强度的变异系 数为 0.06 。几何性质的变异系数取推荐值 0.05 。可以看出, 对于不锈钢冷弯等肢角钢截面, 具有CSM设计承载力函数 的安全分项系数 $\gamma_{M 0}$ 略大于 $\mathrm{EC} 3[3]$ 中推荐的奥氏体不锈
钢的安全分项系数1.10。因此本文建议的不锈钢冷弯等肢 角钢截面承载力的CSM设计公式的安全分项系数可采用 略大于规范推荐值的 1.15 。为与规范推荐值保持一致, 更 精确的CSM设计公式仍在进一步研究中。

表3 不锈钢冷弯等肢角钢截面承载力可靠性分析结果。

\begin{tabular}{llllll}
\hline $\mathbf{n}$ & $\mathbf{K d}, \mathbf{n}$ & $\mathbf{b}$ & $\mathbf{V}_{\boldsymbol{\delta}}$ & $\mathbf{V}_{\mathbf{r}}$ & $\boldsymbol{\gamma}_{\mathbf{M O}}$ \\
\hline 470 & 3.04 & 1.01 & 0.06 & 0.05 & 1.12 \\
\hline
\end{tabular}

\section{8. 结论}

本文介绍了奥氏体不锈钢冷弯等肢角钢短柱的数值 研究。建立并使用可用试验结果验证了非线性有限元模型。 FE技术用于分析具有不同截面几何尺寸及截面长细比的 470 个奥氏体不锈钢冷弯等肢角钢短柱。将得到的承载力 与现行欧洲规范(EC3)及现有CSM设计方法进行比较。

比较结果表明, 现行欧洲规范(EC3)及现有CSM设计 方法在计算奥氏体不锈钢冷弯等肢角钢短柱截面承载力 时过于保守。在确定了现有设计方法需要改进后, 参数研 究得到的FE结果用于修正CSM设计方法, 推荐CSM设计 公式与现有CSM设计方法采用了相同的设计理念。推荐奥 氏体不锈钢冷弯等肢角钢短柱截面承载力的CSM设计公 式计算结果准确且离散性小。此外, 所提出的方法在计算 奥氏体不锈钢冷弯等肢角钢短柱截面承载力时略偏于不 安全, 应采用更高的安全分项系数后应用于设计工作, 本 研究建议采用1.15。奥氏体不锈钢冷弯等肢角钢短柱截面 承载力的更精确CSM设计公式正在研究中。

\section{致谢}

本文为陕西省自然科学基金面上项目《多层柱间支撑 框排架钢结构纵向抗震性能及破坏机理研究》 (2018JM5123)的阶段性成果之一。

\section{参考文献}

[1] SEI/ASCE8-02, Specification for the Design of Cold-Formed Stainless Steel Structural Members [Z], in, American Society of Civil Engineers (ASCE), Reston,2002.

[2] AS/NZS 4673, Cold-formed stainless steel structures [Z], in, Standards Australia,Sydney, 2001.

[3] EN 1993-1-4, Eurocode 3: Design of steel structures - Part 1.4:General rules -supplementary rules for stainless steels [Z], in, European Committee for Standardization(CEN), Brussels, 2006.

[4] CECS 410, Technical specification for stainless steel structures [Z], in, China plan press, Beijing, 2015.

[5] L. Gardner, D.A. Nethercot, Experiments on stainless steel hollow sections-Part 1: material and cross-sectional behaviour [J], J. Constr. Steel Res. 60 (2004) 1291-1318. 
[6] L. Gardner, D. Nethercot, Structural stainless steel design: a new approach [J], Struct. Eng. 82 (2004) 2-28.

[7] L. Gardner, M. Ashraf, Structural design for non-linear metallic materials [J], Eng.Struct. 28 (2006) 926-934.

[8] M. Ashraf, L. Gardner, D.A. Nethercot, Structural stainless steel design: resistance based on deformation capacity [J], J. Struct. Eng. 134 (2008) 402-411.

[9] L. Gardner, The continuous strength method [C], Proc. ICE Struct. Build. 161 (2008) 127-133.

[10] S. Afshan, L. Gardner, The continuous strength method for structural stainless steel design [J], Thin-Walled Struct. 68 (2013) 42-49.

[11] S. Ahmed, M. Ashraf, The Continuous Strength Method for slender stainless steel cross-sections [J], Thin-Walled Struct. 107 (2016) 362-376.

[12] L Zhang, KH Tan, Experimental and numerical studies of fixed-ended cold-formed stainless steel equal-leg angle section columns [J], Eng. Struct. 184 (2019) 134-144.

[13] EN 1993-1-1, Eurocode 3: Design of Steel Structures. Part 1-1: General Rules and Rules for Buildings [Z], Comité Europeo de Normalización, 2005.

[14] M Ashraf, L Gardner, DA Nethercot. Structural stainless steel design: resistance based on deformation capacity [J]. J. Struct. Eng. 134 (2018) 402-411.

[15] A Liew, L Gardner. Ultimate capacity of structural steel cross-sections under compression, bending and combined loading [J]. Structures 1 (2015) 2-11.

[16] C Buchanan, L Gardner, A Liew. The continuous strength method for the design of circular hollow sections [J]. J. Constr. Steel Res. 118 (2016) 207-216.

[17] G Shu, B Zheng, X Shen. Experimental and theoretical study on the behaviour of cold-formed stainless steel stub columns [J]. Int. J. Steel Struct. 13 (2013) 141-153.

[18] O Zhao, B Rossi, L Gardner, B Young. Behaviour of structural stainless steel cross-sections under combined loading - part I: experimental study [J]. Eng. Struct. 89 (2015) 236-246.

[19] O Zhao, B Rossi, L Gardner, B Young. Behaviour of structural stainless steel cross-sections under combined loading - part II: numerical modelling and design approach [J]. Eng. Struct. 89 (2015) 247-259.

[20] O Zhao, B Rossi, L Gardner, B Young. Experimental and numerical studies of ferritic stainless steel tubular cross-sections under combined compression and bending [J]. J. Struct. Eng. 142 (2016) 40-51.
[21] I Arrayago, E Real. Experimental study on ferritic stainless steel RHS and SHS cross-sectional resistance under combined loading [J]. Structures. 4 (2015) 69-79.

[22] ABAQUS, ABAQUS User's Manual v6.11, in, Dassault Systèmes Simulia Corp [M], Providence, RI, USA, 2011.

[23] L. Gardner, D. Nethercot, Numerical modeling of stainless steel structural components-a consistent approach [J], J. Struct. Eng. 130 (2004) 1586-1601.

[24] M. Ashraf, L. Gardner, D.A. Nethercot, Finite element modelling of structural stainless steel cross-sections [J], Thin-Walled Struct. 44 (2006) 1048-1062.

[25] W. Ramberg, W.R. Osgood, Description of stress-strain curves by three parameters [J], Natl. Advis. Comm. Aeronaut. (1943).

[26] K.J.R. Rasmussen, Full-range stress-strain curves for stainless steel alloys [J], J. Constr. Steel Res. 59 (2003) 47-61.

[27] I. Arrayago, E. Real, L. Gardner, Description of stress-strain curves for stainless steel alloys [J], Mater. Des. 87 (2015) $540-552$.

[28] M. Ashraf, L. Gardner, D.A. Nethercot, Strength enhancement of the corner regions of stainless steel cross-sections $[\mathrm{J}], \mathrm{J}$. Constr. Steel Res. 61 (2005) 37-52.

[29] R.G. Dawson, A.C. Walker, Post-buckling of geometrically imperfect plates [J], J. Struct. Div. 98 (1972) 75-94.

[30] H.X. Yuan, Y.Q. Wang, Y.J. Shi, L. Gardner, Residual stress distributions in welded stainless steel sections $[\mathrm{J}]$, Thin-Walled Struct. 79 (2014) 38-51.

[31] R.B. Cruise, L. Gardner, Residual stress analysis of structural stainless steel sections [J], J. Constr. Steel Res. 64 (2008) 352-366.

[32] M. Jandera, L. Gardner, J. Machacek, Residual stresses in cold-rolled stainless steel hollow sections [J], J. Constr. Steel Res. 64 (2008) 1255-1263.

[33] L. Gardner, A New Approach to Structural Stainless Steel Design [D], Department Of Civil And Environment Engineering, Imperial College Of London, London, UK, 2002.

[34] M. Ashraf, Structural Stainless Steel Design: Resistance Based on Deformation Capacity [D], Department Of Civil And Environment Engineering, Imperial College London, UK, 2006.

[35] EN 1990, Eurocode: Basis of Structural Design [Z], European Committee For Standardization (CEN), Brussels, Belgium, 2002.

[36] S. Afshan, P. Francis, N. Baddoo, L. Gardner, Reliability analysis of structural stainless steel design provisions [J], J. Constr. Steel Res. 114 (2015) 293-304. 